\title{
Overall Survival Improved for Contemporary Patients with Melanoma: A 2004-2015 National Cancer Database Analysis
}

\author{
Norma E. Farrow · Megan C. Turner · April K. S. Salama • \\ Georgia M. Beasley
}

Received: April 17, 2020 / Published online: May 28, 2020

(C) The Author(s) 2020

\section{ABSTRACT}

Introduction: Since 2011, encouraging clinical trial results have led to approval of multiple new therapies for advanced melanoma, but the impact of these therapies outside of trial populations is largely unknown. This study examines use of novel therapies and survival in contemporary patients with melanoma.

Methods: Stage I-IV melanoma patients were identified in the 2004-2015 National Cancer Database and grouped into historic (2004-2010) and contemporary (2011-2015) cohorts. Overall survival (OS) was compared using Kaplan-Meier and Cox proportional hazard modeling adjusting for patient, tumor, and facility characteristics.

Norma E. Farrow and Megan C. Turner contributed equally to the completion of this study.

Digital Features To view digital features for this article go to https://doi.org/10.6084/m9.figshare.12280856.

N. E. Farrow $(\varangle) \cdot$ M. C. Turner · G. M. Beasley Department of Surgery, Duke University Medical Center, Durham, NC, USA

e-mail: norma.farrow@duke.edu

A. K. S. Salama

Department of Medicine, Duke University Medical

Center, Durham, NC, USA
Results: Of 268,668 patients, 136,828 were classified as historic and 131,840 as contemporary. Among all stages, immunotherapy utilization was significantly higher among contemporary patients $(5.3 \%$ vs. $5.1 \%$, $p=0.006$ ). Adjusted OS was improved in the contemporary cohort (hazard ratio [HR]: 0.90 $p<0.001)$. There was no difference in OS among stage I/II patients between groups (HR: $0.99, p=0.63)$, while OS was significantly improved for contemporary stage III/IV patients (HR: 0.85, $p<0.001$ ). Among stage III/IV patients who received immunotherapy, OS was improved for the contemporary cohort (HR: $0.87, p=0.014)$.

Conclusions: Adjusted overall survival for contemporary melanoma patients is improved. This effect is driven by improvements for those with advanced stage disease, particularly those that received immunotherapy and BRAF/MEK targeted therapies.

Keywords: Immunotherapy; Melanoma; Metastatic melanoma; Survival outcomes; Targeted therapy 


\section{Key Summary Points}

The prognosis for patients with advanced melanoma has historically been poor. However, advancements in treatment for melanoma have been rapid in the last decade, with multiple new therapies being approved since 2011 including immune therapies and targeted therapies.

This study examines rates of novel therapy usage for melanoma and assesses changes in overall survival over time in patients with melanoma using the National Cancer Database.

Overall survival for patients with melanoma has improved among patients diagnosed after 2011, driven by improvements in survival for patients with stage III/IV disease.

Improvements in survival are likely due to novel systemic therapies such as immune therapies and targeted therapies, first approved for use in advanced melanoma in 2011.

\section{INTRODUCTION}

From 1991 to 2005, the incidence of melanoma increased by $140 \%$ in the USA, faster than any other cancer [1]. Estimates of new melanoma cases approached 100,000 in 2018 , with a lifetime risk of $2.6 \%$ ( 1 in 38 ) of being diagnosed with melanoma for Caucasians [1]. While the majority of people diagnosed with melanoma are over age 55 , the incidence of melanoma among children, adolescents, and young adults has more than doubled over the last 4 decades with young females at highest risk for the deadly cancer [2]. As the incidence of melanoma continues to increase, effective treatment strategies for patients with every stage of disease are needed.
Localized excision for thin melanoma can be curative in up to $90 \%$ of cases [3]. However, prior to 2011, the prognosis for patients with locally advanced or metastatic melanoma was dismal. Starting in 2011, a wave of new therapies revolutionized the treatment of metastatic melanoma [4-11]. Examples of novel approaches approved in recent years include immune checkpoint blockade (anti-CTLA-4, anti-PD-1), targeted therapies (BRAF inhibition/MEK inhibition, BRAFi/MEKi) and oncolytic viral therapy with talimogene laherparepvec (TVEC) $[7,8,12]$. In appropriately designed randomized controlled trials, compared with historic therapies, both immune checkpoint blockade and targeted therapies have demonstrated improvements for patients with advanced melanoma in both disease-free and overall survival $[7,13]$.

Although there have been recent dramatic changes in melanoma treatment strategies based on favorable clinical trial results, clinical trial populations are not always reflective of a generalized population. Additionally, the pace of integrating novel approved therapy into generalized practice is rarely studied; limitations of novel therapy may include cost, side effects, or lack of treating institution resources. Given recent FDA approval of multiple therapies for melanoma, and promising recent national statistics showing overall improvements in mortality for melanoma, we hypothesize that usage of immune therapy has increased since 2011 and that contemporary patients with advanced melanoma have improved survival compared with patients prior to 2011 [14]. Here, we aim to examine the rates of novel therapy usage and whether improvements in overall survival have been observed in a sample of contemporary melanoma patients.

\section{METHODS}

The Duke University Institutional Review Board provided an exemption for this retrospective review of patients diagnosed with melanoma in the National Cancer Database (NCDB). Patients $\geq 18$ years of age diagnosed with pathologic American Joint Committee on Cancer (AJCC, Version 6 or 7 based on year of 
diagnosis) stage I-IV melanoma between 2004 and 2015 were identified in the NCDB. The NCDB is a clinical oncology database that was developed and is maintained by The American Cancer Society in conjunction with The Commission on Cancer (CoC) of the American College of Surgeons. Standardized data on treatment, outcomes, and demographics are extracted by certified registrars in over 1500 CoC-accredited centers [15]. Taken together, these data include over $70 \%$ of all newly diagnosed cancer cases in the US.

Year of diagnosis was used to create historic (2004-2010) and contemporary (2011-2015) cohorts. Demographic variables including patient age, gender, race, annual income, education, and insurance type and status were extracted. Median education and income level were identified by ZIP code of patient residence and determined as above or below the median. Facility type was categorized as community (accession 100-500 new cancer cases/year), comprehensive community (accession $>500$ new cancer cases per year), or academic centers (accession $>500$ new cancer cases per year and participating in postgraduate medical education and research activities). Facility location is a regional designation. Clinical and oncologicspecific factors assessed included comorbidity [categorized by Charlson-Deyo (CD) score], pathologic stage of the tumor, location of the tumor (extremities, head/neck, trunk), and surgical margins [16]. While the NCDB does not provide drug-specific data, immune therapy is classified in the NCDB as "biological or chemical agents that alter the immune system or change the host's response to tumor cells," including modern agents such as immune checkpoint inhibitors and talimogene laherparepvec (TVEC), historic agents such as interferon-alpha and interleukins, and investigational agents such as vaccines, oncolytic viruses, and Toll-like receptor agonists, among others. Targeted therapy (BRAFi/MEKi) is classified as chemotherapy in the NCDB. In addition to overall survival, postoperative outcomes including length of stay, 30-day mortality, 90-day mortality, and readmission were assessed. Disease-free survival is not a metric captured in the NCDB.
To determine if contemporary melanoma management strategies, approved in or after 2011, were associated with improved overall survival, Kaplan-Meier unadjusted survival curves were generated comparing cohorts of patients diagnosed from 2004-2010 and 2011-2015. Comparisons were generated for the entire stage I-IV cohort, early stage I/II, and late stage III/IV.

Multivariable logistic regression models adjusting for patient factors (age, gender, race, insurance coverage, income, education, and CD score), tumor characteristics (stage, location, margin positivity), and facility characteristics (academic vs. comprehensive community vs. community hospital, facility location) were constructed to compare overall survival between the cohorts while controlling for known confounders. Cox proportional hazard models were then used to analyze the association between cohorts (2004-2010 and 2011-2015) and overall survival. Comparisons were generated for the cohort at large (stage I-IV), early stage I/II, late stage III/IV, as well as a subgroup analysis for patients with stage III/IV disease who received immunotherapy.

All statistical tests were two-tailed and considered to be significant at $p<0.05$. Analyses were performed using R Version 3.4.0 (Vienna, Austria).

\section{RESULTS}

Of the 268,668 patients identified with stage I-IV melanoma in the NCDB 2004-2015, 136,828 were diagnosed between 2004-2010 (historic), and 131,840 were diagnosed between 2011-2015 (contemporary). The number of patients diagnosed with melanoma increased and nearly doubled from 2004 to 2015, with 16,520 diagnosed cases in 2004 and 29,047 in 2015. Age at diagnosis was older in the contemporary cohort (median 63 years, interquartile range [IRQ]: 51-73) compared with the historic cohort (median 59 years, IQR: 47-72, $p<0.001)$. Comorbidity scores were higher in those diagnosed in 2011-2015 (CD score $>0$ : $15 \%$ vs. $11.3 \%, p<0.001$ ), and patients in the contemporary group were diagnosed at higher 
Table 1 Baseline characteristics by historic (2004-2010) and contemporary (2011-2015) cohorts

\begin{tabular}{|c|c|c|c|c|}
\hline & $\begin{array}{l}2004-2010 \\
(N=136,828)\end{array}$ & $\begin{array}{l}2011-2015 \\
(N=131,840)\end{array}$ & $\begin{array}{l}\text { Combined } \\
(N=268,668)\end{array}$ & $P$ value \\
\hline \multicolumn{5}{|l|}{ Patient characteristics } \\
\hline Median age, years (IQR) & $59(47.72)$ & $63(51.73)$ & $61(49.72)$ & $<0.001$ \\
\hline Gender & & & & $<0.001$ \\
\hline Male & $77,819(56.9 \%)$ & $77,014(58.4 \%)$ & $154,833(57.6 \%)$ & \\
\hline Female & $59,009(43.1 \%)$ & $54,826(41.6 \%)$ & $113,835(42.4 \%)$ & \\
\hline Race & & & & $<0.001$ \\
\hline White & $133,175(98.8 \%)$ & $128,820(98.6 \%)$ & $261,995(98.7 \%)$ & \\
\hline Black & $784(0.6 \%)$ & $742(0.6 \%)$ & $1526(0.6 \%)$ & \\
\hline Other & $876(0.6 \%)$ & $1055(0.8 \%)$ & $1931(0.7 \%)$ & \\
\hline Income level & & & & $<0.001$ \\
\hline Below median & $30,651(23.3 \%)$ & $28,739(22.6 \%)$ & $59,390(22.9 \%)$ & \\
\hline Above median & $100,855(76.7 \%)$ & $98,698(77.4 \%)$ & $199,553(77.1 \%)$ & \\
\hline Insurance status & & & & $<0.001$ \\
\hline None & $3516(2.6 \%)$ & $3042(2.3 \%)$ & $6558(2.5 \%)$ & \\
\hline Private & $78,373(58.7 \%)$ & $68,271(52.7 \%)$ & $146,644(55.7 \%)$ & \\
\hline Government & $51,727(38.7 \%)$ & $58,135(44.9 \%)$ & $109,862(41.8 \%)$ & \\
\hline $\begin{array}{l}\text { Charlson-Deyo comorbidity } \\
\text { score }\end{array}$ & & & & $<0.001$ \\
\hline 0 & $121,327(88.7 \%)$ & $112,117(85.0 \%)$ & $233,444(86.9 \%)$ & \\
\hline 1 & $13,033(9.5 \%)$ & $15,936(12.1 \%)$ & $28,969(10.8 \%)$ & \\
\hline 2 & $1911(1.4 \%)$ & $2837(2.2 \%)$ & $4748(1.8 \%)$ & \\
\hline 3 & $557(0.4 \%)$ & $950(0.7 \%)$ & $1507(0.6 \%)$ & \\
\hline \multicolumn{5}{|l|}{ Tumor characteristics } \\
\hline AJCC 7 pathologic stage & & & & $<0.001$ \\
\hline I & $5482(10.3 \%)$ & $3245(6.2 \%)$ & $8727(8.3 \%)$ & \\
\hline II & $23,001(43.2 \%)$ & $22,790(43.6 \%)$ & $45,791(43.4 \%)$ & \\
\hline III & $18,686(35.1 \%)$ & $19,967(38.2 \%)$ & $38,653(36.6 \%)$ & \\
\hline IV & $6031(11.3 \%)$ & $6301(12.0 \%)$ & $12,332(11.7 \%)$ & \\
\hline Tumor location & & & & $<0.001$ \\
\hline Extremities & $60,662(44.3 \%)$ & $57,589(43.7 \%)$ & $118,251(44.0 \%)$ & \\
\hline Head and neck & $27,630(20.2 \%)$ & $27,819(21.1 \%)$ & $55,449(20.6 \%)$ & \\
\hline Trunk & $43,527(31.8 \%)$ & $40,859(31.0 \%)$ & $84,386(31.4 \%)$ & \\
\hline
\end{tabular}


Table 1 continued

\begin{tabular}{lllll}
\hline & $\begin{array}{l}\mathbf{2 0 0 4 - 2 0 1 0} \\
(\boldsymbol{N}=\mathbf{1 3 6 , 8 2 8})\end{array}$ & $\begin{array}{l}\mathbf{2 0 1 1 - 2 0 1 5} \\
(\boldsymbol{N}=\mathbf{1 3 1 , 8 4 0})\end{array}$ & $\begin{array}{l}\text { Combined } \\
(\boldsymbol{N}=\mathbf{2 6 8}, \mathbf{6 6 8})\end{array}$ & P value \\
\hline $\begin{array}{l}\text { Unknown or overlapping } \\
\text { location }\end{array}$ & $5009(3.7 \%)$ & $5573(4.2 \%)$ & $10,582(3.9 \%)$ & \\
$\begin{array}{l}\text { Treatment characteristics } \\
\text { Facility type }\end{array}$ & & & \\
Community & $7815(7.4 \%)$ & $6806(6.4 \%)$ & $14,621(6.9 \%)$ & \\
Comprehensive & $44,206(41.7 \%)$ & $39,787(37.3 \%)$ & $83,993(39.5 \%)$ & \\
Academic & $53,944(50.9 \%)$ & $59,974(56.3 \%)$ & $113,918(53.6 \%)$ & \\
Postoperative chemotherapy & & & & \\
No & $93,317(93.1 \%)$ & $122,975(94.0 \%)$ & $216,292(93.6 \%)$ & \\
Yes & $6867(6.9 \%)$ & $7875(6.0 \%)$ & $14,742(6.4 \%)$ & \\
Immunotherapy (includes stage & & & & \\
I-IV) & & $124,819(94.7 \%)$ & $254,680(94.8 \%)$ & \\
No & $129,861(94.9 \%)$ & $7021(5.3 \%)$ & $13,988(5.2 \%)$ & \\
Yes & $6967(5.1 \%)$ & & & \\
\hline
\end{tabular}

$A J C C$ American Joint Commission on Cancer, $I Q R$ interquartile range

pathologic stages (stage III/IV: $50.2 \%$ vs. $46.4 \%$, $p<0.001$ ). Patients were more frequently treated at academic institutions in the contemporary cohort $(56.5 \%$ vs. $50.9 \%, p<0.001)$, were less likely to be covered by private insurance $(52.7 \%$ vs. $58.7 \%, p<0.001)$, and were more likely to be covered by government insurance (44.9\% vs. $38.7 \%, p<0.001)$ compared with the historic cohort (Table 1). While statistically significant, differences between tumor location and margin positivity were not clinically different between cohorts.

There were 12,051 patients included in a subset analysis of stage III/IV melanoma patients who received immune therapy, 5765 in the 2004-2010 cohort, and 6286 in the 2011-2015 cohort (Table 2). Patients receiving immune therapy in the contemporary cohort were older (median 55 years, IQR: 45-65 vs. 51 years, IQR: $40-60, p<0.001)$, with higher comorbidty scores (CD score $>0$ : $15.5 \%$ vs. $9.9 \%, p<0.001)$, and diagnosed at more advanced pathologic stage (stage IV: $26.6 \%$ vs.
$11.8 \%, p<0.001)$. More patients in the contemporary cohort had government insurance, and fewer had private insurance, compared with the historic cohort (government: $34.2 \%$ vs. $21.1 \%$; private: $61.5 \%$ vs. $73.7 \%, p<0.001$ ). The majority of patients in each cohort were treated at academic institutions, though the percentage of patients treated at academic institutions increased in the contemporary cohort (academic: $59.9 \%$ vs. $55.3 \%, p<0.001$ ). The groups were similar in terms of race and income level, and, while statistically significant, differences between gender were not clinically significant.

Our first aim was to examine rates of administration of novel therapies. The percent of patients overall (stage I-IV) who received immune therapy was statistically significantly higher in the contemporary cohort for all patients (stage I-IV, $5.3 \%$ vs. $5.1 \%, p<0.006$ ). The percentage of stage III/IV patients receiving immune therapy in $2015(27.8 \%)$ increased compared with prior years, and the total 
Table 2 Characteristics of subset of patients with stage III/IV melanoma receiving immune therapy by historic (2004-2010) and contemporary (2011-2015) cohorts

\begin{tabular}{|c|c|c|c|c|}
\hline & $\begin{array}{l}2004-2010 \\
(N=5765)\end{array}$ & $\begin{array}{l}2011-2015 \\
(N=6286)\end{array}$ & $\begin{array}{l}\text { Combined } \\
(N=12,051)\end{array}$ & $P$ value \\
\hline \multicolumn{5}{|l|}{ Patient characteristics } \\
\hline Median age, years (IQR) & $51(40.60)$ & $55(45.65)$ & $53(42.63)$ & $<0.001$ \\
\hline Gender & & & & 0.008 \\
\hline Male & $3434(59.6 \%)$ & $3893(61.9 \%)$ & $7327(60.8 \%)$ & \\
\hline Female & $2331(40.4 \%)$ & $2393(38.1 \%)$ & $4724(39.2 \%)$ & \\
\hline Race & & & & 0.106 \\
\hline White & $5624(98.3 \%)$ & $6091(97.7 \%)$ & $11,715(98.0 \%)$ & \\
\hline Black & $45(0.8 \%)$ & $60(1.0 \%)$ & $105(0.9 \%)$ & \\
\hline Other & $54(0.9 \%)$ & $81(1.3 \%)$ & $135(1.1 \%)$ & \\
\hline Income level & & & & 0.394 \\
\hline Below median & $1345(24.4 \%)$ & $1525(25.0 \%)$ & $2870(24.7 \%)$ & \\
\hline Above median & $4175(75.6 \%)$ & $4563(75.0 \%)$ & $8738(75.3 \%)$ & \\
\hline Insurance status & & & & $<0.001$ \\
\hline None & $293(5.2 \%)$ & $265(4.3 \%)$ & $558(4.7 \%)$ & \\
\hline Private & $4184(73.7 \%)$ & $3808(61.5 \%)$ & $7992(67.4 \%)$ & \\
\hline Government & $1199(21.1 \%)$ & $2114(34.2 \%)$ & $3313(27.9 \%)$ & \\
\hline $\begin{array}{l}\text { Charlson-Deyo comorbidity } \\
\text { score }\end{array}$ & & & & $<0.001$ \\
\hline 0 & $5192(90.1 \%)$ & $5318(84.6 \%)$ & $10,510(87.2 \%)$ & \\
\hline 1 & $514(8.9 \%)$ & $790(12.6 \%)$ & $1304(10.8 \%)$ & \\
\hline 2 & $46(0.8 \%)$ & $143(2.3 \%)$ & $189(1.6 \%)$ & \\
\hline 3 & $13(0.2 \%)$ & $35(0.6 \%)$ & $48(0.4 \%)$ & \\
\hline \multicolumn{5}{|l|}{ Tumor characteristics } \\
\hline AJCC pathologic stage & & & & $<0.001$ \\
\hline III & $5086(88.2 \%)$ & $4616(73.4 \%)$ & $9702(80.5 \%)$ & \\
\hline IV & $679(11.8 \%)$ & $1670(26.6 \%)$ & $2349(19.5 \%)$ & \\
\hline Tumor location & & & & $<0.001$ \\
\hline Extremities & $2311(40.1 \%)$ & $2129(33.9 \%)$ & $4440(36.8 \%)$ & \\
\hline Head and neck & $774(13.4 \%)$ & $894(14.2 \%)$ & $1668(13.8 \%)$ & \\
\hline Trunk & $2087(36.2 \%)$ & $1951(31.0 \%)$ & $4038(33.5 \%)$ & \\
\hline $\begin{array}{l}\text { Unknown or overlapping } \\
\text { location }\end{array}$ & $593(10.3 \%)$ & $1312(20.9 \%)$ & $1905(15.8 \%)$ & \\
\hline
\end{tabular}


Table 2 continued

\begin{tabular}{llll}
$2004-2010$ & $2011-2015$ & Combined & $P$ value \\
$(N=5765)$ & $(N=6286)$ & $(N=12,051)$ & \\
\hline
\end{tabular}

Treatment characteristics

Facility type

Community

Comprehensive

Academic

Postoperative chemotherapy

No

Yes

$$
\begin{aligned}
& 263(6.7 \%) \\
& 1486(38.0 \%) \\
& 2161(55.3 \%)
\end{aligned}
$$

$265(6.2 \%)$

3997 (93.8\%)
$278(5.9 \%)$

$1602(34.2 \%)$

$2811(59.9 \%)$

$896(14.3 \%)$

$5383(85.7 \%)$
$541(6.3 \%)$

3088 (35.9\%)

4972 (57.8\%)

$<0.001$

$1161(11.0 \%)$

$9380(89.0 \%)$ number of patients receiving immune therapy is increasing each year (747 patients total in 2004, 1057 in 2011, 1634 in 2015) (Fig. 1). Receipt of postoperative therapies showed a decrease in postoperative chemotherapies between the cohorts with $6.0 \%$ of stage I-IV patients in the 2011-2015 cohort receiving chemotherapy compared with $6.9 \%$ in the 2004-2010 cohort $(p<0.001)$. Additionally, in the subset of stage III/IV melanoma patients receiving immunotherapy, fewer patients in the contemporary cohort received postoperative chemotherapy $(85.7 \%$ vs. $93.8 \%, p<0.001)$ (Table 2).

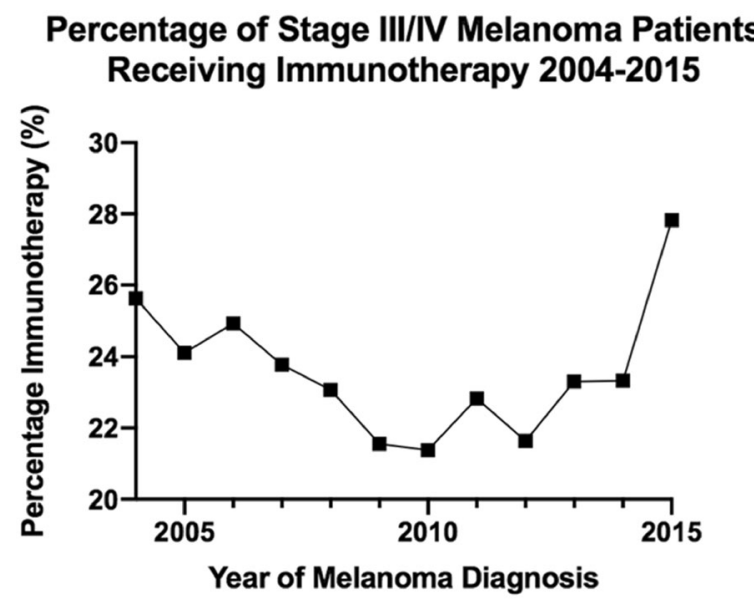

Fig. 1 Percentage of stage III/IV melanoma patients receiving immune therapy by year
Our second aim was to examine survival outcomes between the two cohorts. Early mortality was equivalent between the cohorts at $0.2 \%$ 30-day mortality and $0.7 \%$ 90-day mortality for each. In aggregate, unadjusted survival from melanoma (all stages) is slightly worse in the cohort diagnosed between 2011-2015 compared with those diagnosed between 2004-2010 (Fig. 2a). For patients with early stage disease (stage I/II), unadjusted survival was slightly worse in the contemporary cohort (Fig. 2b). However, improved unadjusted survival is seen in the contemporary cohort for late stages, III/IV (Fig. 2c).

In a subsequent analysis of all stage melanoma patients in both cohorts adjusting for patient demographic, tumor and facility characteristics, we found that patients with higher CD comorbidity scores have worse overall survival compared with those with $\mathrm{CD}$ scores of 0 (hazard ratio [HR]: 1.22 for CD of $1,1.86$ for CD of 2 , and 2.00 for $\mathrm{CD}$ of $3, p<0.001$ ) (Table 3 ). Interestingly, women with melanoma have improved overall survival relative to men [HR: 0.81, 95\% confidence interval (CI): 0.78-0.84]. Black patients have worse survival (HR: 1.26, 95\% CI 1.10-1.45) relative to white patients. Those who are insured with private (HR: 0.61, 95\% CI 0.56-0.67) or government (HR: 0.72, 95\% CI 0.66-0.79) insurance have improved survival relative to the uninsured $(p<0.001)$. Patients with head and neck tumors (HR: 1.23, 


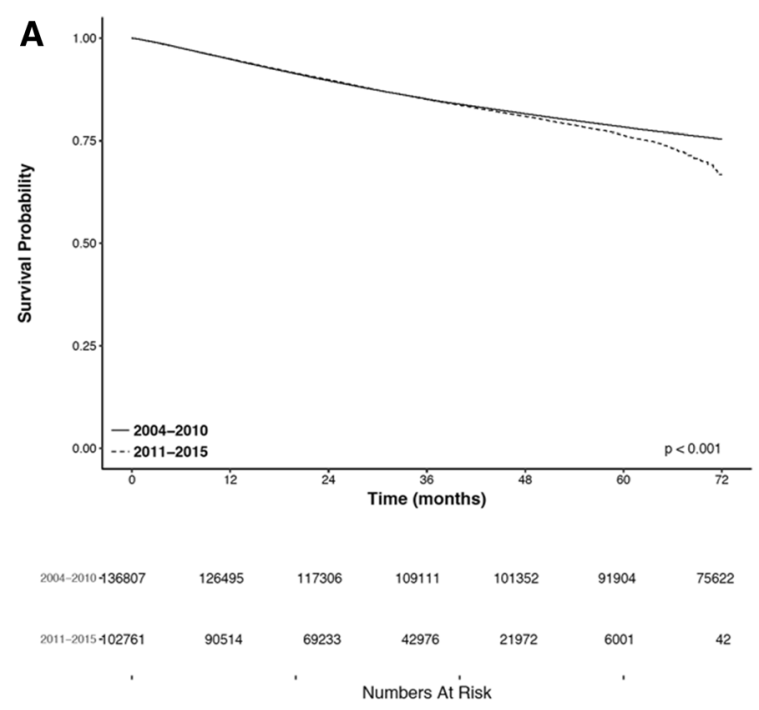

4Fig. 2 a Unadjusted survival curves for patients with stage I-IV melanoma by historical (2004-2010) and contemporary (2011-2015) cohorts. b Unadjusted survival curves for patients with stage I-II melanoma by historical (2004-2010) and contemporary (2011-2015) cohorts. c Unadjusted survival curves for patients with stage III-IV melanoma by historical (2004-2010) and contemporary (2011-2015) cohorts

95\% CI 1.18-1.27) or trunk tumors (HR: 1.18, 95\% CI: 1.14-1.23) have worse survival relative to those with extremity tumors (all $p<0.001$ ). Patients treated at academic institutions have improved adjusted overall survival relative to community programs (HR: 0.86, CI: 0.81-0.91, $p<0.001)$. Stage III patients have improved adjusted survival compared with those with stage IV disease (HR: 0.38, CI: 0.36-0.40, $p<0.001)$; a similar increased improvement in survival was seen among stage III patients who received immunotherapy compared with stage IV patients who received immunotherapy (HR: 0.31, CI:0.26-0.36, $p<0.001$ ) (Table 4).

In the overall adjusted survival analyses, patients diagnosed with stage I-IV melanoma from 2011-2015 have a 10\% reduced mortality compared with those who were diagnosed between 2004-2010 (HR: 0.90, 95\% CI $0.88-0.95, p<0.001)$. Among early stage disease (stage I-II) patients, no difference is seen in overall survival (HR: 0.99, CI 0.94-1.04, $p=0.63$ ). For late stage disease (stage III/IV), a $15 \%$ reduction in mortality is observed in the 2011-2015 cohort compared with the 2004-2010 cohort (HR: 0.85, CI 0.81-0.88, $p<0.001)$. For the subset of patients with stage III/IV melanoma receiving immunotherapy, a $13 \%$ overall survival improvement is seen in patients diagnosed between 2011-2015 compared with those from 2004-2010 (HR: 0.87, CI 0.79-0.97, $p=0.014$ ) (Table 4).

\section{DISCUSSION}

Our study shows an overall improvement in adjusted survival for patients with melanoma diagnosed after 2011 compared with prior to 
Table 3 Predictors of survival for patients with stage I-IV melanoma

\begin{tabular}{|c|c|c|c|}
\hline & Hazard ratio & 95\% confidence interval (lower, upper) & $P$ value \\
\hline $2011-2015$ cohort $($ ref $=2010-2014$ cohort $)$ & 0.90 & $(0.88,0.94)$ & $<0.001$ \\
\hline Age (per decade) & 1.04 & $(1.04,1.04)$ & $<0.001$ \\
\hline Female sex & 0.81 & $(0.78,0.84)$ & $<0.001$ \\
\hline \multicolumn{4}{|l|}{ Race (ref = White) } \\
\hline Black & 1.26 & $(1.10,1.45)$ & 0.001 \\
\hline Other & 1.22 & $(1.04,1.44)$ & 0.016 \\
\hline Income above median & 0.88 & $(0.86,0.91)$ & $<0.001$ \\
\hline \multicolumn{4}{|l|}{ Insurance status (ref = uninsured) } \\
\hline Private & 0.61 & $(0.56,0.67)$ & $<0.001$ \\
\hline Government & 0.72 & $(0.66,0.79)$ & $<0.001$ \\
\hline \multicolumn{4}{|l|}{ Facility type (ref = community) } \\
\hline Comprehensive community program & 0.95 & $(0.90,1.01)$ & 0.075 \\
\hline Research/academic program & 0.86 & $(0.81,0.91)$ & $<0.001$ \\
\hline \multicolumn{4}{|l|}{ Charlson-Deyo comorbidity score $(\mathrm{ref}=0)$} \\
\hline 1 & 1.22 & $(1.17,1.27)$ & $<0.001$ \\
\hline 2 & 1.86 & $(1.72,2.00)$ & $<0.001$ \\
\hline 3 & 2.00 & $(1.75,2.29)$ & $<0.001$ \\
\hline \multicolumn{4}{|l|}{ AJCC stage (ref = stage IV) } \\
\hline I & 0.09 & $(0.08,0.10)$ & $<0.001$ \\
\hline II & 0.19 & $(0.18,0.20)$ & $<0.001$ \\
\hline III & 0.38 & $(0.36,0.40)$ & $<0.001$ \\
\hline \multicolumn{4}{|l|}{ Tumor location (ref $=$ extremities) } \\
\hline Head and neck & 1.23 & $(1.18,1.27)$ & $<0.001$ \\
\hline Trunk & 1.18 & $(1.14,1.23)$ & $<0.001$ \\
\hline Unknown or overlapping location & 1.14 & $(1.00,1.29)$ & 0.045 \\
\hline Positive surgical margins & 1.57 & $(1.48,1.65)$ & $<0.001$ \\
\hline
\end{tabular}

2011 using a large, contemporary clinical data set. This finding is particularly encouraging given that patients in the 2011-2015 cohort were older, had higher comorbidity scores, and were diagnosed with melanoma at more advanced stages. The improvements in adjusted overall survival appear to be largely explained by improvements in overall survival for patients with AJCC stage III/IV disease while there was no change in survival for patients with stage I/II disease. Treatment for stage I/II disease has remained relatively unchanged since 1991, although more patients are now being diagnosed at earlier time points with stage III disease 
Table 4 Predictors of survival for patients with stage III/IV melanoma who received immunotherapy

\begin{tabular}{|c|c|c|c|}
\hline & Hazard ratio & 95\% Confidence interval (lower, upper) & $P$ value \\
\hline $2011-2015$ cohort $($ ref $=2010-2014$ cohort $)$ & 0.87 & $(0.77,0.97)$ & 0.014 \\
\hline Age (per decade) & 1.01 & $(1.01,1.02)$ & $<0.001$ \\
\hline Female sex & 0.75 & $(0.68,0.84)$ & $<0.001$ \\
\hline \multicolumn{4}{|l|}{ Race (ref $=$ white) } \\
\hline Black & 1.44 & $(0.86,2.42)$ & 0.168 \\
\hline Other & 1.50 & $(0.99,2.28)$ & 0.056 \\
\hline Income above median & 0.87 & $(0.78,0.97)$ & 0.011 \\
\hline \multicolumn{4}{|l|}{ Insurance status (ref $=$ uninsured) } \\
\hline Private & 0.71 & $(0.57,0.88)$ & 0.002 \\
\hline Government & 0.93 & $(0.73,1.17)$ & 0.518 \\
\hline \multicolumn{4}{|l|}{ Facility type (ref $=$ community $)$} \\
\hline Comprehensive community program & 1.00 & $(0.82,1.21)$ & 0.99 \\
\hline Research/academic program & 0.95 & $(0.78,1.14)$ & 0.558 \\
\hline \multicolumn{4}{|l|}{ Charlson-Deyo comorbidity score $(\mathrm{ref}=0)$} \\
\hline 1 & 1.18 & $(1.03,1.36)$ & 0.019 \\
\hline 2 & 2.38 & $(1.75,3.26)$ & $<0.001$ \\
\hline 3 & 2.02 & $(1.04,3.91)$ & 0.037 \\
\hline \multicolumn{4}{|l|}{ AJCC stage (ref = stage IV) } \\
\hline III & 0.31 & $(0.27,0.36)$ & $<0.001$ \\
\hline \multicolumn{4}{|l|}{ Tumor location (ref $=$ extremities) } \\
\hline Head and neck & 1.09 & $(0.94,1.26)$ & 0.253 \\
\hline Trunk & 1.15 & $(1.03,1.27)$ & 0.012 \\
\hline Unknown or overlapping location & 1.14 & $(0.77,1.69)$ & 0.517 \\
\hline Positive surgical margins & 1.58 & $(1.30,1.90)$ & $<0.001$ \\
\hline
\end{tabular}

given the increased use of sentinel lymph node biopsy [17]. Our study supports that the prognosis for patients with stage III/IV melanoma nationally has improved since 2011.

Given that the improvement in overall survival was seen for advanced stage melanoma patients and not those with early stage disease, the improvement is likely the result of novel systemic therapies including immune checkpoint blockade and targeted therapy (BRAFi/ MEKi). This finding is supported by the recent analysis by Tella et al., which showed improved survival among stage III and IV melanoma patients treated at the highest volume national centers in the same time frame as our study [18]. Their analysis, which also used the NCDB, found that receipt of immunotherapy was associated with improved survival in both stage III and IV patients and found a significantly improved median survival for those patients with stage IV disease managed at the highest tercile centers after 2011 (9.8 vs. 13 months, 
$p<0.001$ ) [18]. While they found no difference in receipt of chemotherapy or immunotherapy for stage III patients between low- and highvolume centers, likely as a result of modern adjuvant therapies not being approved until the end of the study period, they did find that stage IV patients treated at high-volume centers were more likely to receive systemic treatment with chemotherapy or immunotherapy. Our study shows that that a higher proportion of contemporary patients are now receiving care at academic institutions and that treatment at academic institutions is associated with improved survival among overall melanoma patients. Our findings complement those of Tella et al. and may reflect more patients being treated at multi-disciplinary, high-volume academic institutions where modern immune and targeted therapies may be more readily available, including on clinical trials. However, our data suggest that this improvement in survival is not limited to high-performing, multi-disciplinary centers given the improvements in the contemporary cohorts after adjustment for facility type. Given this, both studies support that the use of systemic novel therapies accounted for much of the improvements in overall survival.

In our study, we found an adjusted 15\% reduction in mortality observed in stage III/IV melanoma patients in the contemporary cohort compared with the historic cohort (HR: 0.85, CI $0.81-0.88, p<0.001)$, while the subset of patients with stage III/IV disease receiving immunotherapy had a 13\% improvement in overall survival in patients diagnosed between 2011-2015 compared with those from 2004-2010 (HR: 0.87, CI 0.79-0.97, $p=0.014$ ). This suggests that contemporary immune therapy is more effective than that used historically. Furthermore, the proportion of patients receiving immune therapy with stage IV disease increased significantly between the two cohorts (11.8\% to $26.6 \%, p<0.001)$, suggesting that more patients with stage IV disease are benefiting from these therapies being approved for use in metastatic disease and not just in the setting of unresectable stage III disease or in the adjuvant setting. Notably, two recent NCDB studies evaluating patients with stage III and IV melanoma separately found improvements in overall survival in more contemporary cohorts (through 2013 for stage III and through 2011 for stage IV) $[19,20]$. Both studies, like ours, had limited follow-up time after approval of novel therapies but similarly found improvements in survival for both stage III and IV disease. Importantly, the first modern immune therapy was not approved for use in the adjuvant setting until the end of our study period (anti-CTLA4 in 2015, anti-PD1 in 2017 and BRAFi/MEKi in 2018), which suggests that patients with stage III disease received benefit in these studies from systemic therapies used in clinical trials or as treatment for metastatic disease in the setting of recurrence [19]. Given promising clinical trial results for both immune checkpoint inhibitors and targeted therapies, as well as additional approvals for these therapies in both the metastatic and adjuvant settings since the end of our study period, we expect that benefits of systemic novel therapies will continue to improve in future studies.

The use of targeted therapy with BRAFi/MEKi is captured in the NCDB as chemotherapy, which makes it difficult to discern trends in its use over the study duration and to differentiate it from more historic forms of chemotherapy such as darcarbazine. Given the limitations of our data set, we are unable to make any direct attribution of the improvement in overall survival seen in our study to BRAFi/MEKi. However, given promising clinical trial results for targeted therapy, and the relatively high rates of "chemotherapy" use in the subset of patients in our trial who received immune therapies, we expect that BRAFi/MEKi also contributed to the improvements in survival we saw in our trial. Supporting this, a smaller retrospective study with drug-specific data available recently evaluated patients with metastatic melanoma in Denmark and found that patients diagnosed in 2016 had improved outcomes compared with those diagnosed in 2014 or 2012 as well as increasing use of both anti-PD-1 and BRAF/ MEKi therapy [21]. Their results support our findings that the use of immune therapy and targeted therapies has led to improved overall survival. 
Our findings that receipt of immunotherapy was nearly equivalent, though statistically different, at $5.3 \%$ for the contemporary stage I-IV cohort compared with $5.1 \%$ for the historical cohort $(p=0.006)$ were somewhat surprising. However, multiple immune therapies have been used historically in melanoma including interferon-alpha and interleukin-2, with limited effectiveness. It is important to note that though the overall use of immune therapy was low, about half of each cohort was patients with stage I and II disease, and therefore immunotherapy would not have been indicated for this population. Of the remaining patients, the majority were stage III; given the first approval of modern immune therapy in the adjuvant setting was not granted until 2015, these patients also would not have been expected to receive immune therapy other than in the setting of clinical trials or recurrence. In the 2015 cohort, $27.8 \%$ of patients with stage III/IV melanoma received immune therapy, and we would expect this rate to continue to increase as therapies are approved and adopted into practice. Ipilimumab was the first therapy for advanced melanoma to demonstrate an improvement in overall survival in a randomized phase III trial and received FDA approval in 2011. While response rates were modest, clear long-term survivors exist [22-25]. More effective therapies targeting the PD-1 pathway were approved as second-line therapy in 2014 $[26,27]$. However, nivolumab and pembrolizumab, as well as the combination of ipilimumab and nivolumab, did not receive designations as first-line therapy for metastatic melanoma until late 2015, likely limiting their use even in our contemporary cohort [28, 29]. However, patients receiving immune therapies on clinical trials are included in the NCDB and would therefore be included in our analysis. Given the approval of these therapies for advanced and metastatic melanoma, as well as the more recent approvals for use in the adjuvant setting, and the now widespread use of immune checkpoint inhibitors in a number of other malignancies leading to increasing provider comfort with these drugs, we would expect the rate of immunotherapy use to continue to increase beyond our study period. As these therapies enter wider use and are used for broader indications, we hope this will lead to continued improvements in survival in contemporary patients.

Our findings are subject to several limitations. The NCDB is a validated data set, but is limited in the granularity of clinical variables, is subject to selection bias, and therefore is subject to unmeasured confounding. The large number of patients captured in this data set helps mitigate the effects of the bias in the analysis adjusting for patient, tumor, and facility characteristics. However, missing data, which were excluded from the analysis, and lack of granularity regarding the specific type, sequence, and duration of therapies as well as lack of information on tumor markers within the data sets limit our ability to determine if the survival effect is in fact due to these advancements in clinical care of melanoma. However, these limitations are equally distributed between the two cohorts so the comparison of survival between the two allows for confident determination of effect size. The large cohort of melanoma patients captured in the NCDB is reflective of contemporary oncology practices in the US population, permits adequate adjustment for known covariates, and lends our results considerable external validity.

\section{CONCLUSION}

Using a NCDB data set of 268,668 melanoma patients, we demonstrated that overall survival for patients with stage III/IV melanoma from 2011-2015 is improved compared with patients from 2004-2010. This improvement appears to be largely related to novel immune therapies, the first of which received FDA approval in 2011. Our study suggests that immune therapy use is increasing but may be underutilized in patients with advanced melanoma. As these novel therapies enter wider use, ongoing efforts to optimize patient selection and efforts to discover biologic strategies to overcome resistance to these therapies can further improve prognosis for patients with advanced melanoma. 


\section{ACKNOWLEDGEMENTS}

Funding. No funding or sponsorship was received for this study or publication of this article. The Rapid Service Fee was funded by the authors.

Authorship. All named authors meet the International Committee of Medical Journal Editors (ICMJE) criteria for authorship for this article, take responsibility for the integrity of the work as a whole, and have given their approval for this version to be published.

Authorship Contributions. Georgia M. Beasley and Megan C. Turner conceived of the presented study; Megan C. Turner performed the statistical analysis; Megan C. Turner, Georgia M. Beasley and Norma E. Farrow verified and interpreted the statistical methods and results. All authors contributed to writing, editing, and providing feedback on the final manuscript. Norma E. Farrow and Megan C. Turner contributed equal effort to the study as first authors.

Prior Presentation. This study was previously presented as a poster at the Society of Surgical Oncology's 72nd Annual Cancer Symposium in March 2019 in San Diego, CA.

Disclosures. The authors report no proprietary or commercial interest in any product mentioned or concept discussed in this article. Georgia M. Beasley was a consultant for Cardinal Health and receives clinical trial support from Istari Oncology. April K.S. Salama receives research funding (paid to institution) from Bristol Myers Squibb, Celldex, Dynavax, Genentech, Immunocore, Merck, and Reata and is a consultant for Array and Merck. Norma E. Farrow is supported by a National Institutes of Health T-32 grant 5T32CA093245 in surgical oncology and a research grant from the Polka Dot Mama Melanoma Foundation. Megan C. Turner has nothing to disclose.

Compliance with Ethics Guidelines. The Duke University Institutional Review Board provided an exemption for this retrospective review of patients diagnosed with melanoma in the National Cancer Database (NCDB). Authors had permission to access this database.

Data Availability. The National Cancer Data Base (NCDB) is a joint project of the Commission on Cancer (CoC) of the American College of Surgeons and the American Cancer Society. The CoC's NCDB and the hospitals participating in the CoC NCDB are the source of the de-identified data used herein; they have not verified and are not responsible for the statistical validity of the data analysis or the conclusions derived by the author.

Open Access. This article is licensed under a Creative Commons Attribution-NonCommercial 4.0 International License, which permits any non-commercial use, sharing, adaptation, distribution and reproduction in any medium or format, as long as you give appropriate credit to the original author(s) and the source, provide a link to the Creative Commons licence, and indicate if changes were made. The images or other third party material in this article are included in the article's Creative Commons licence, unless indicated otherwise in a credit line to the material. If material is not included in the article's Creative Commons licence and your intended use is not permitted by statutory regulation or exceeds the permitted use, you will need to obtain permission directly from the copyright holder. To view a copy of this licence, visit http://creativecommons.org/licenses/by$\mathrm{nc} / 4.0 /$.

\section{REFERENCES}

1. American Cancer Society. Key Statistics in Melanoma Skin Cancer. https://www.cancer.org/cancer/ melanoma-skin-cancer/about/key-statistics.html.

2018. https://www.cancer.org/cancer/melanomaskin-cancer/about/key-statistics.html. Accessed 19 Nov 2018.

2. Mitsis DKL, Groman A, Beaupin LM, Salerno KE, Francescutti V, Skitzki JJ, et al. Trends in demographics, incidence, and survival in children, adolescents and young adults (AYA) with melanoma: a surveillance, epidemiology and end results (SEER) 
population-based analysis. J Clin Oncol. 2015;33: 9058.

3. Max Fullah M, Viola F, Wiel BVD, Klop WMC, Józwiak K, Wouters MWJM, et al. External validation of the 8th edition melanoma staging system of the American Joint Committee on Cancer (AJCC): effect of adding EORTC sentinel node (SN) tumor burden criteria on prognostic accuracy in stage III. J Clin Oncol. 2018;36:9500.

4. Bollag G, Hirth P, Tsai J, Zhang J, Ibrahim PN, Cho $\mathrm{H}$, et al. Clinical efficacy of a RAF inhibitor needs broad target blockade in BRAF-mutant melanoma. Nature. 2010;467(7315):596-9. https://doi.org/10. 1038/nature09454.

5. Flaherty KT, Infante JR, Daud A, Gonzalez R, Kefford RF, Sosman J, et al. Combined BRAF and MEK inhibition in melanoma with BRAF V600 mutations. N Engl J Med. 2012;367(18):1694-703. https://doi.org/10.1056/NEJMoa1210093.

6. D'Angelo SP, Larkin J, Sosman JA, Lebbe C, Brady B, Neyns B, et al. Efficacy and safety of nivolumab alone or in combination with ipilimumab in patients with mucosal melanoma: a pooled analysis. J Clin Oncol. 2017;35(2):226-35. https://doi. org/10.1200/jco.2016.67.9258.

7. Hodi FS, Chesney J, Pavlick AC, Robert C, Grossmann KF, McDermott DF, et al. Combined nivolumab and ipilimumab versus ipilimumab alone in patients with advanced melanoma: 2-year overall survival outcomes in a multicentre, randomised, controlled, phase 2 trial. Lancet Oncol. 2016;17(11):1558-68. https://doi.org/10.1016/ s1470-2045(16)30366-7.

8. Andtbacka RH, Kaufman HL, Collichio F, Amatruda $\mathrm{T}$, Senzer N, Chesney J, et al. Talimogene laherparepvec improves durable response rate in patients with advanced melanoma. J Clin Oncol. 2015;33(25):2780-8. https://doi.org/10.1200/jco. 2014.58.3377.

9. Franklin C, Livingstone E, Roesch A, Schilling B, Schadendorf D. Immunotherapy in melanoma: recent advances and future directions. Eur J Surg Oncol. 2017;43(3):604-11. https://doi.org/10.1016/ j.ejso.2016.07.145.

10. Eigentler TK, Gutzmer R, Hauschild A, Heinzerling L, Schadendorf D, Nashan D, et al. Adjuvant treatment with pegylated interferon alpha- 2 a versus low-dose interferon alpha-2a in patients with highrisk melanoma: a randomized phase III DeCOG trial. Ann Oncol. 2016;27(8):1625-32. https://doi. org/10.1093/annonc/mdw225.

11. Ascierto PA, Minor D, Ribas A, Lebbe C, O'Hagan A, Arya N, et al. Phase II trial (BREAK-2) of the BRAF inhibitor dabrafenib (GSK2118436) in patients with metastatic melanoma. J Clin Oncol. 2013;31(26): 3205-11. https://doi.org/10.1200/JCO.2013.49. 8691.

12. Long GV, Stroyakovskiy D, Gogas H, Levchenko E, de Braud F, Larkin J, et al. Combined BRAF and MEK inhibition versus BRAF inhibition alone in melanoma. N Engl J Med. 2014;371(20):1877-88. https://doi.org/10.1056/NEJMoa1406037.

13. Long GV, Flaherty KT, Stroyakovskiy D, Gogas H, Levchenko E, de Braud F, et al. Dabrafenib plus trametinib versus dabrafenib monotherapy in patients with metastatic BRAF V600E/K-mutant melanoma: long-term survival and safety analysis of a phase 3 study. Ann Oncol. 2017;28(7):1631-9. https://doi.org/10.1093/annonc/mdx176.

14. Siegel RL, Miller KD, Jemal A. Cancer statistics, 2020. CA Cancer J Clin. 2020;70(1):7-30. https:// doi.org/10.3322/caac. 21590 .

15. Raval MV, Bilimoria KY, Stewart AK, Bentrem DJ, Ko CY. Using the NCDB for cancer care improvement: an introduction to available quality assessment tools. J Surg Oncol. 2009;99(8):488-90. https://doi.org/10.1002/jso.21173.

16. Deyo RA, Cherkin DC, Ciol MA. Adapting a clinical comorbidity index for use with ICD-9-CM administrative databases. J Clin Epidemiol. 1992;45(6): 613-9.

17. Morton DL, Thompson JF, Cochran AJ, Mozzillo N, Nieweg OE, Roses DF, et al. Final trial report of sentinel-node biopsy versus nodal observation in melanoma. N Engl J Med. 2014;370(7):599-609. https://doi.org/10.1056/NEJMoa1310460.

18. Tella SH, Kommalapati A, Ganti AK, Marr AS. Association between hospital volume, therapy types, and overall survival in stage III and IV cutaneous malignant melanoma. J Natl Comprehens Cancer Netw JNCCN. 2019;17(11):1334-422. https://doi.org/10.6004/jnccn.2019.7320.

19. Song Y, Tieniber AD, Gimotty PA, Mitchell TC, Amaravadi RK, Schuchter LM, et al. Survival outcomes of patients with clinical stage III melanoma in the era of novel systemic therapies. Ann Surg Oncol. 2019;26(13):4621-30. https://doi.org/10. 1245/s10434-019-07599-y.

20. Sinnamon AJ, Neuwirth MG, Gimotty PA, Gangadhar TC, Amaravadi RK, Schuchter LM, et al. Association of first-in-class immune checkpoint inhibition and targeted therapy with survival in patients with stage IV melanoma. JAMA Oncol. 2018;4(1):126-8. jamaoncol.2017.3462. 
21. Donia M, Ellebaek E, Heide Ollegaard T, Duval L, Bull Aaby J, Heojberg L, et al. The real-world impact of moder treatments on the survival of patients with metastatic melanoma. Eur J Cancer. 2019;108: 25-322.

22. Maio M, Grob JJ, Aamdal S, Bondarenko I, Robert C, Thomas L, et al. Five-year survival rates for treatment-naive patients with advanced melanoma who received ipilimumab plus dacarbazine in a phase III trial. J Clin Oncol. 2015;33(10):1191-6. https://doi. org/10.1200/JCO.2014.56.6018.

23. Schadendorf D, Hodi FS, Robert C, Weber JS, Margolin $\mathrm{K}$, Hamid $\mathrm{O}$, et al. Pooled analysis of longterm survival data from phase II and phase III trials of ipilimumab in unresectable or metastatic melanoma. J Clin Oncol. 2015;33(17):1889-944. https:// doi.org/10.1200/JCO.2014.56.2736.

24. Hodi FS, O'Day SJ, McDermott DF, Weber RW, Sosman JA, Haanen JB, et al. Improved survival with ipilimumab in patients with metastatic melanoma. N Engl J Med. 2010;363(8):711-23. https:// doi.org/10.1056/NEJMoa1003466.

25. McDermott D, Haanen J, Chen TT, Lorigan P, O'Day S, Investigators MDX. Efficacy and safety of ipilimumab in metastatic melanoma patients surviving more than 2 years following treatment in a phase III trial (MDX010-20). Ann Oncol. 2013;24(10):2694-8. https://doi.org/10.1093/ annonc/mdt291.

26. Weber J, Mandala M, Del Vecchio M, Gogas HJ, Arance AM, Cowey CL, et al. Adjuvant nivolumab versus ipilimumab in resected stage III or IV melanoma. N Engl J Med. 2017;377(19):1824-35. https://doi.org/10.1056/NEJMoa1709030.

27. Schachter J, Ribas A, Long GV, Arance A, Grob JJ, Mortier L, et al. Pembrolizumab versus ipilimumab for advanced melanoma: final overall survival results of a multicentre, randomised, open-label phase 3 study (KEYNOTE-006). Lancet. 2017;390(10105):1853-62. https://doi.org/10.1016/ S0140-6736(17)31601-X.

28. Keytruda (pembrolizumab) [package insert]. Merck Sharp \& Dohme Corp., Whitehouse Station, NJ. 2018.

29. Opdivo (nivolumab) [package insert]. Bristol-Myers Squibb Company., Princeton, NJ. 2018. 